\title{
40-Gb/s All-Optical Packet Switching With a Distributed-Feedback Laser as All-Optical Flip-Flop
}

\author{
Koen Huybrechts, Student Member, IEEE, Takuo Tanemura, Member, IEEE, Yoshiaki Nakano, Member, IEEE, \\ Roel Baets, Fellow, IEEE, and Geert Morthier, Senior Member, IEEE
}

\begin{abstract}
All-optical flip-flops (AOFFs) have recently received increased attention as elements for all-optical packet-switched networks. In this letter, we use a single off-the-shelf distributedfeedback laser as AOFF to switch 40-Gb/s packets with a guard time as low as $150 \mathrm{ps}$.
\end{abstract}

Index Terms-All-optical flip-flop (AOFF), distributed-feedback laser (DFB), optical packet switching.

\section{INTRODUCTION}

$\mathbf{T}$ HE increasing demand for network traffic will create a technological bottleneck on future optical routing systems. New internet-based services are gaining more public attention and will increase the need for higher bit rates. However, the router forwarding capacity does not match the fiber bandwidth at these high bit rates. The opto-electronic and electrooptic conversions $(\mathrm{O} / \mathrm{E} / \mathrm{O})$ needed to process data in the network nodes are extremely power consuming and require expensive electronic circuits. Moreover, most electrooptic switches have rather slow switching times (more than $1 \mathrm{~ns}$ ). Transparent networks based on all-optical packet switching offer a solution since all the processing is done completely in the optical layer [1]-[4].

All-optical flip-flops (AOFFs) are important building blocks in the implementation of all-optical packet switching because they can act as optical memory elements which store the header information while the output of the flip-flop is directly used to route the payload directly to the correct output port without any $\mathrm{O} / \mathrm{E} / \mathrm{O}$ conversions [2]. Recently, we demonstrated that a single antireflection-coated distributed-feedback (DFB) laser with a $\lambda / 4$-shift operates as a fast AOFF under the injection of continuous-wave light [5], [6]. DFB lasers are already widely used in today's telecommunication industry and the added functionality as optical memory element offers, therefore, an economically viable way to achieve all-optical packet switching.

Manuscript received December 19, 2008; revised January 26, 2009. First published March 16, 2009; current version published May 08, 2009. This work was supported by the Fund for Scientific Research (FWO), by the IAP-project "Photonics@be," and by the Erasmus Mundus program. The work of K. Huybrechts was supported by the Institute for the Promotion of Innovation through Science and Technology (IWT) under a specialization grant.

K. Huybrechts, R. Baets, and G. Morthier are with the Photonics Research Group, Department of Information Technology (INTEC), Ghent University-IMEC, B-9000 Ghent, Belgium (e-mail: Koen.Huybrechts@intec.UGent.be; baets@intec.ugent.be; morthier@intec.ugent.be).

T. Tanemura and Y. Nakano are with the Research Centre for Advanced Science and Technology (RCAST), University of Tokyo, Meguro-ku, Tokyo, Japan (e-mail: tanemura@ee.t.u-tokyo.ac.jp; nakano@ee.t.u-tokyo.ac.jp).

Color versions of one or more of the figures in this letter are available online at http://ieeexplore.ieee.org

Digital Object Identifier 10.1109/LPT.2009.2016671
In this letter, we demonstrate all-optical packet switching with a DFB laser as AOFF and a Mach-Zehnder interferometer semiconductor optical amplifier (MZI-SOA) as a $40-\mathrm{Gb} / \mathrm{s}$ wavelength converter. The addition of a label at the end of the packets allows the use of packets with a variable length. We obtain packet switching with a guard time of only $150 \mathrm{ps}$, which is the best reported guard time for all-optical packet switching experiments so far to the authors' knowledge.

\section{CONCEPT}

In current networks, the optical packets are wavelengthdivision multiplexed (WDM) on different optical carriers to exploit the fiber bandwidth. These carriers can be created by an array of DFB laser diodes, each emitting light at one channel of the WDM grid. In electronic configurations, the packets are demultiplexed and converted to electrical signals by an array of photodiodes. After processing in the electric layer, the signals are converted back to one of the optical carriers of the WDM grid.

Recently, we demonstrated that a single DFB laser diode can act as a broadband AOFF under the injection of continuouswave light [6]. By using an array of DFB lasers where each laser has a different lasing wavelength matched to the WDM grid, one can switch a laser in the array ON and OFF simply by injecting an optical pulse. Such an optical pulse can originate from an all-optical header processor unit which compares the header with a predefined bit-sequence [7]. Using the scheme of Fig. 1, we can switch ON the DFB laser that matches the header routing information. A wavelength converter can be applied to convert the packet to the wavelength of the DFB laser. An arrayed waveguide grating (AWG) can subsequently switch the packets to different output ports according to their new wavelength. At the end of the packet, we insert a reset pulse to switch OFF the laser again. This allows to use packets of variable length. When using packets with a fixed length, the set-pulse generated by the optical header processor can be delayed over a fiber delay line.

The technique illustrated here allows very fast switching compared to electronic switches. In a separate experiment with higher pulse energies $(2.2 \mathrm{pJ})$, switching times of $45 \mathrm{ps}$ were observed [8]. Even more important is the advantage that DFB lasers are already a mature technology and that they can be easily matched with the WDM grid. The device can work under broadband operation: the only restriction on the wavelength of the pulses and holding beam is that they should be outside the stopband of the DFB grating. The disadvantage of the necessity of a holding beam for the flip-flop operation in a DFB laser can be addressed by distributing one single holding beam over all the lasers of the array. The devices under study are dependent on the polarization of the pulses. In theory, optimized designs 


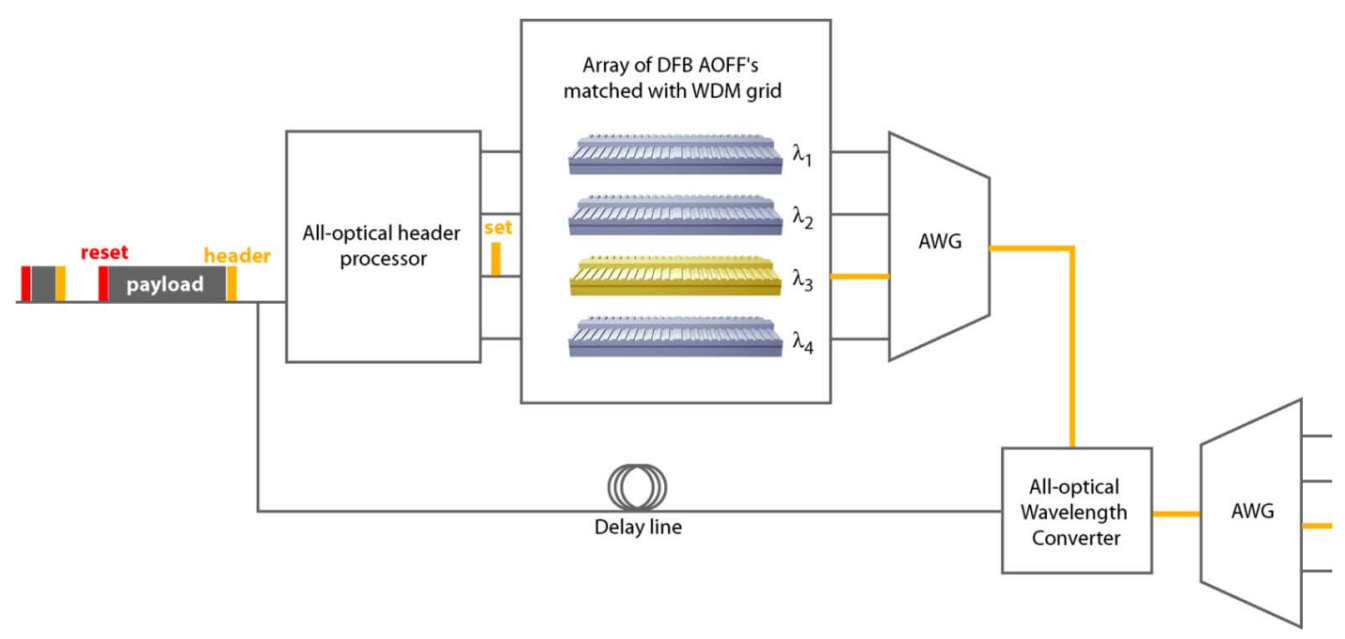

Fig. 1. Concept for all-optical packet switching based on an array of DFB lasers with different wavelengths. The two AWGs can be identical.

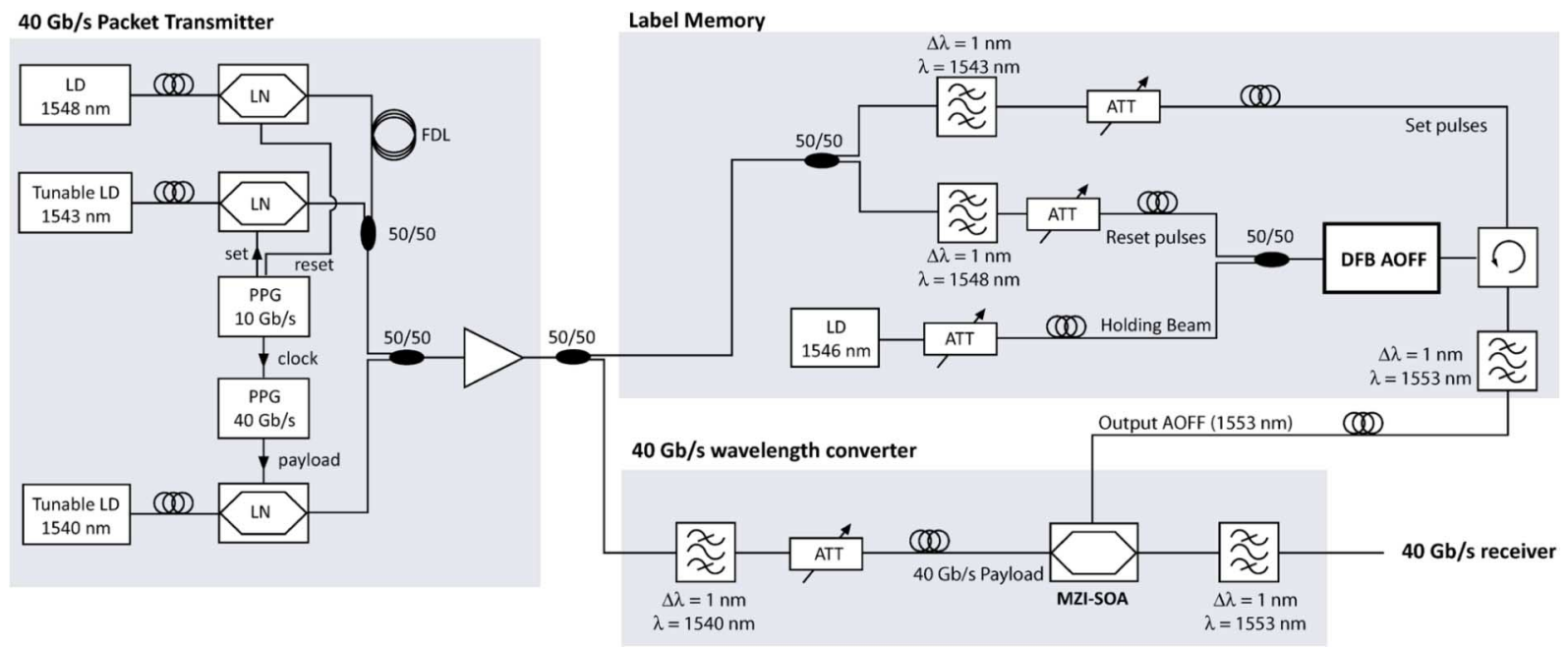

Fig. 2. Schematic of the measurement setup for optical packet switching with a DFB AOFF. (LD: laser diode; ATT: attenuator; FDL: fiber delay line; PPG: pulse pattern generator; LN: lithium niobate modulator.)

for the laser section could be used that are insensitive to the polarization.

\section{EXPERIMENT}

To demonstrate the concept, we use one single DFB laser as AOFF to switch $40-\mathrm{Gb} / \mathrm{s}$ packets with a variable duration (see Fig. 2). The 40-Gb/s nonreturn-to-zero (NRZ) payload $(1540 \mathrm{~nm})$ is combined with label-encoded pulses having a duration of $150 \mathrm{ps}$. To use packets with a variable duration, we add a pulse at the start (set-pulses at $1543 \mathrm{~nm}$ ) and end (reset-pulses at $1548 \mathrm{~nm}$ ) of the packet. After amplification of this signal through an erbium-doped fiber amplifier, the set-pulses and reset-pulses are extracted seperately by an optical filter. The reset-pulses are combined with a holding beam $(1546 \mathrm{~nm})$ of approximately $3 \mathrm{dBm}$ to make the DFB laser operative in the bistable regime. The set-pulses are injected at the opposite side of the laser to restore the uniformity of the carriers [5]. The output of the DFB AOFF is sent to an SOA-based MZI which converts the optical packet to the wavelength of the AOFF $(1553 \mathrm{~nm})$. The MZI-SOA consists of an optimized active-layer structure to reduce the carrier lifetime, enabling $40-\mathrm{Gb} / \mathrm{s} \mathrm{NRZ}$ wavelength conversion without push-pull operation [9].
The extracted set- and reset-pulses are depicted in Fig. 3 together with the corresponding output of the AOFF and the switched $40-\mathrm{Gb} / \mathrm{s}$ packets. The packets have a length of 140 and $40 \mathrm{~ns}$. The pulse energies can be as low as $200 \mathrm{fJ}$, but the flip-flop has faster switching times when using higher pulse energies. The transient behaviour of the switched packets is shown in Fig. 4. In this case, pulse energies of $500 \mathrm{fJ}$ are used and one can see that a guard time of only 150 ps should be taken into account. In a different experiment, switching times as low as $45 \mathrm{ps}$ were observed when using pulses of $2.2 \mathrm{pJ}$. Error-free operation can be obtained as illustrated in the bit-error-rate (BER) curve of Fig. 5. The power penalty of $2.4 \mathrm{~dB}$ is mostly due to the wavelength conversion.

\section{CONCLUSION}

A fast and practical method is demonstrated to switch 40-Gb/s optical packets using an off-the-shelf DFB laser as AOFF. Packets with a variable duration can be switched by adding an extra label at the end of the packet. A guard time of 150 ps has been achieved. In the future, the DFB laser array should be integrated on a single chip together with the all-optical header processor, wavelength converter, and AWGs. Such 

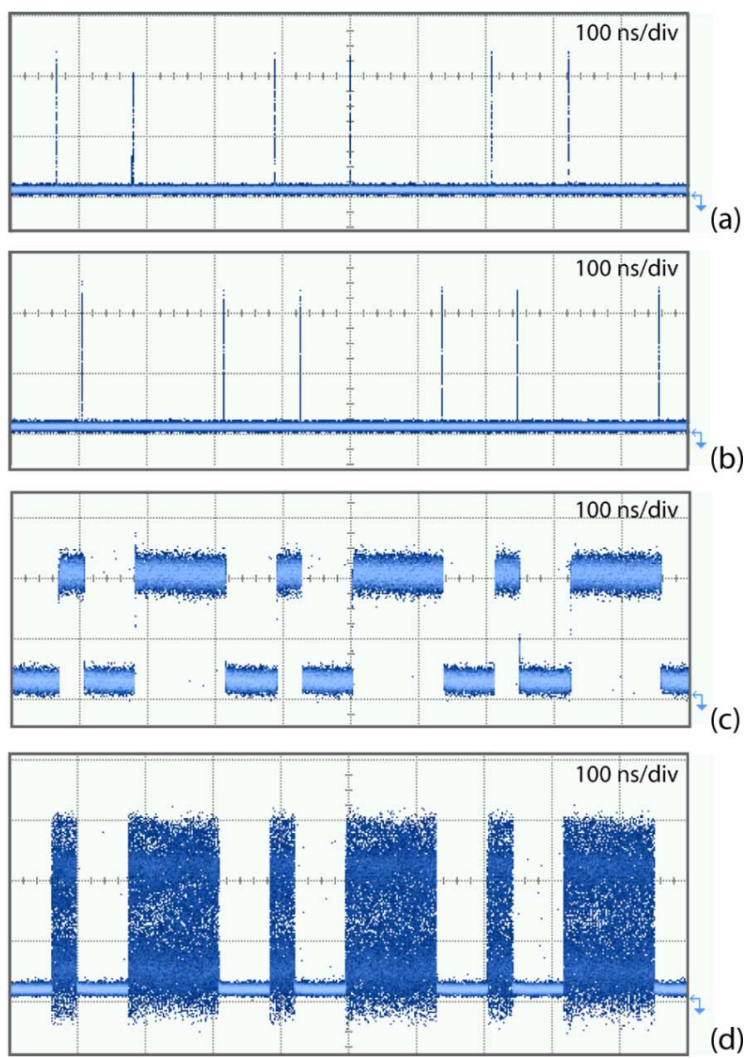

(c)

Fig. 3. Results for all-optical packet-switching with a DFB AOFF. (a) Setpulses; (b) reset-pulses; (c) output of DFB AOFF; (d) optically switched packets.
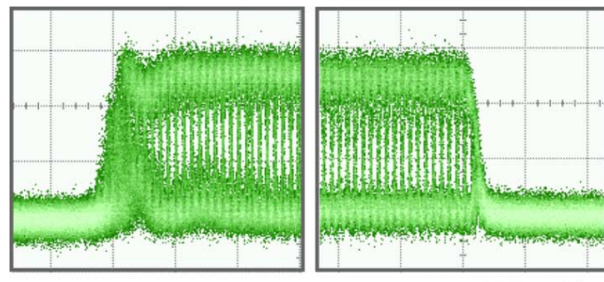

$200 \mathrm{ps} / \mathrm{div}$
Fig. 4. Transient behaviour at the start and end of the switched packets.

a chip would have a great potential for a compact integrated all-optical packet switching circuit.

\section{ACKNOWLEDGMENT}

The authors would like to thank Alcatel-Thales III/V-Laboratories for providing the DFB laser diodes and Mitsubishi Electric for the MZI-SOA wavelength converter.

\section{REFERENCES}

[1] J. Herrera, O. Raz, E. Tangdiongga, Y. Liu, H. C. H. Mulvad, F. Ramos, J. Marti, G. Maxwell, A. Poustie, M. T. Hill, H. de Waardt, G. D. Khoe, A. M. J. Koonen, and H. J. S. Dorren, "160-Gb/s all-optical packet switching over a 110-km field installed optical fiber link," J. Lightw. Technol., vol. 26, no. 1, pp. 176-182, Jan. 1, 2008.

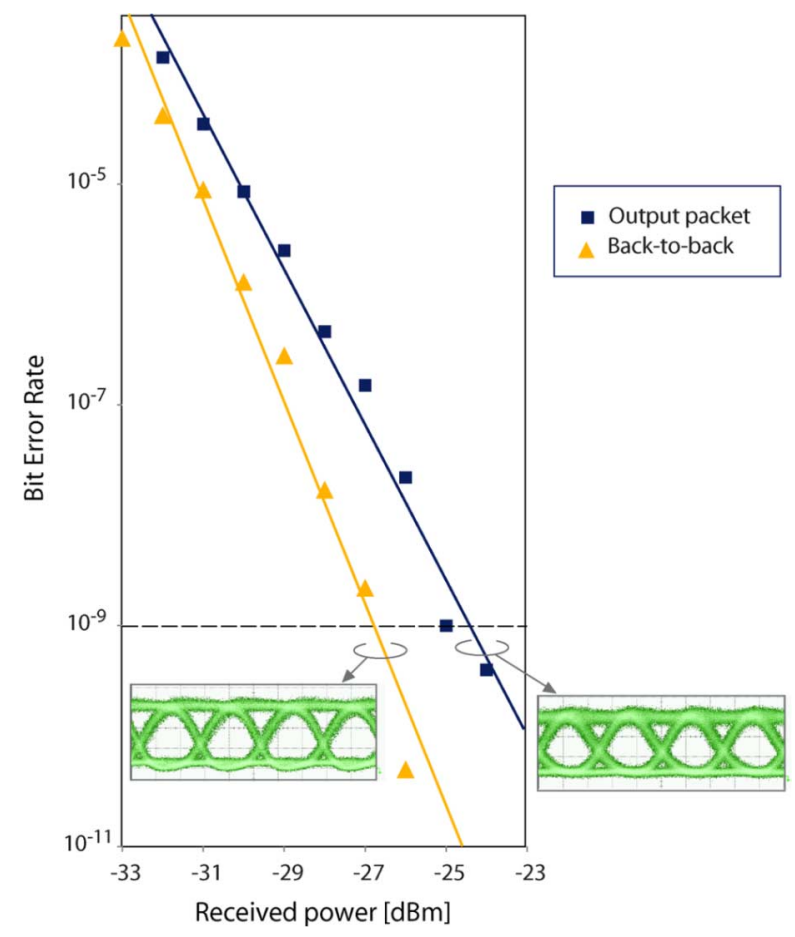

Fig. 5. BER measurements for the back-to-back and output.

[2] H. J. S. Dorren, M. T. Hill, Y. Liu, N. Calabretta, A. Srivatsa, F. M. Huijskens, H. de Waardt, and G. D. Khoe, "Optical packet switching and buffering by using all-optical signal processing methods," J. Lightw. Technol., vol. 21, no. 1, pp. 2-12, Jan. 2003.

[3] N. Calabretta, H. Jung, J. Herrera, E. Tangdiongga, A. Koonen, and H. Dorren, " $1 \times 4$ all-optical packet switch at $160 \mathrm{~Gb} / \mathrm{s}$ employing optical processing of scalable in-band address labels," presented at the Optical Fiber Communication Conf. (OFC/NFOEC), San Diego, CA, 2008, Postdeadline paper 33.

[4] M. Takenaka, K. Takeda, Y. Kanema, and Y. Nakano, "All-optical switching of $40 \mathrm{~Gb} / \mathrm{s}$ packets by MMI-BLD optical label memory," Opt. Express, vol. 14, no. 22, pp. 10785-10789, 2006.

[5] K. Huybrechts, W. D'Oosterlinck, G. Morthier, and R. Baets, "Proposal for an all-optical flip-flop using a single distributed feedback laser diode," IEEE Photon. Technol. Lett., vol. 20, no. 1, pp. 18-20, Jan. 1, 2008.

[6] K. Huybrechts, G. Morthier, and R. Baets, "Fast all-optical flip-flop based on a single distributed feedback laser diode," Opt. Express, vol. 16, no. 15, pp. 11405-11410, 2008.

[7] I. Kang, M. Rasras, M. Dinu, L. Buhl, S. Cabot, M. Cappuzzo, L. Gomez, Y. Chen, S. Patel, C. Giles, N. Dutta, A. Piccirilli, and J. Jaques, "Latency-free all-optical recognition of 32-bit optical bit patterns at $40 \mathrm{~Gb} / \mathrm{s}$ using a passive optical correlator," presented at the Eur. Conf. Optical Communication (ECOC), Brussels, Belgium, 2008, Paper th.3.E.6.

[8] K. Huybrechts, R. Baets, and G. Morthier, "Improved switching times for a distributed feedback all-optical flip-flop," unpublished.

[9] T. Hatta, T. Miyahara, Y. Miyazaki, K. Takagi, K. Matsumoto, T. Aoyagi, K. Motoshima, K. Mishina, A. Maruta, and K. Kitayama, "Polarization-insensitive monolithic 40-Gbps SOA-MZI wavelength converter with narrow active waveguides," IEEE J. Sel. Topics Quantum Electron., vol. 13, no. 1, pp. 32-39, Jan./Feb. 2007. 